\title{
Metformin Protects H9C2 Cardiomyocytes from High-Glucose and Hypoxia/Reoxygenation Injury via Inhibition of Reactive Oxygen Species Generation and Inflammatory Responses: Role of AMPK and JNK
}

\author{
Mingyan Hu, Ping Ye, Hua Liao, Manhua Chen, and Feiyan Yang \\ Department of Cardiology, Central Hospital of Wuhan, Tongji Medical College, Huazhong University of Science and Technology, \\ Wuhan 430014, China
}

Correspondence should be addressed to Feiyan Yang; feiyanyang@hotmail.com

Received 28 December 2015; Revised 8 February 2016; Accepted 29 March 2016

Academic Editor: Chin-Moi Chow

Copyright $\odot 2016$ Mingyan Hu et al. This is an open access article distributed under the Creative Commons Attribution License, which permits unrestricted use, distribution, and reproduction in any medium, provided the original work is properly cited.

\begin{abstract}
Metformin is a first-line drug for the management of type 2 diabetes. Recent studies suggested cardioprotective effects of metformin against ischemia/reperfusion injury. However, it remains elusive whether metformin provides direct protection against hypoxia/reoxygenation (H/R) injury in cardiomyocytes under normal or hyperglycemic conditions. This study in H9C2 rat cardiomyoblasts was designed to determine cell viability under $\mathrm{H} / \mathrm{R}$ and high-glucose $(\mathrm{HG}, 33 \mathrm{mM})$ conditions and the effects of cotreatment with various concentrations of metformin $(0,1,5$, and $10 \mathrm{mM})$. We further elucidated molecular mechanisms underlying metformin-induced cytoprotection, especially the possible involvement of AMP-activated protein kinase (AMPK) and Jun $\mathrm{NH}(2)$-terminal kinase (JNK). Results indicated that $5 \mathrm{mM}$ metformin improved cell viability, mitochondrial integrity, and respiratory chain activity under HG and/or H/R $(P<0.05)$. The beneficial effects were associated with reduced levels of reactive oxygen species generation and proinflammatory cytokines (TNF- $\alpha$, IL-1 $\alpha$, and IL-6) $(P<0.05)$. Metformin enhanced phosphorylation level of AMPK and suppressed HG + H/R induced JNK activation. Inhibitor of AMPK (compound C) or activator of JNK (anisomycin) abolished the cytoprotective effects of metformin. In conclusion, our study demonstrated for the first time that metformin possessed direct cytoprotective effects against $\mathrm{HG}$ and H/R injury in cardiac cells via signaling mechanisms involving activation of AMPK and concomitant inhibition of JNK.
\end{abstract}

\section{Introduction}

Diabetes mellitus is associated with a number of long-term complications, including nephropathy, retinopathy, stroke, and cardiovascular diseases, which lead to decreased quality of life and reduced life expectancy [1]. Patients with type 2 diabetes mellitus (T2DM) have a higher risk for coronary heart disease [2] and are more susceptible to myocardial ischemia/reperfusion (I/R) injury as compared with nondiabetic individuals $[3,4]$. To date, no agent is in routine clinical use to protect the myocardium against I/R injury, although several pharmacological agents have been studied with respect to their ability to attenuate $I / R$ injury [5]. Metformin (1,1-dimethylbiguanide), a biguanide derivate, is the most widely prescribed drug in the treatment of T2DM [6]. Clinical trials demonstrated that metformin reduced diabetes-related death and all-cause mortality $[7,8]$ and previous exploratory studies suggested that metformin had direct vascular beneficial effects, for example, in a murine model of myocardial $\mathrm{I} / \mathrm{R}$, but the underlying mechanisms of this beneficial effect are not completely understood $[9,10]$.

The pathogenesis of hypoxia/reoxygenation (H/R) injury (a major component of $I / R$ injury) in diabetic hearts is associated with cardiomyocyte apoptosis [11] and overproduction of reactive oxygen species (ROS) [12]. It is widely accepted that metformin leads to activation of AMP-activated protein kinase (AMPK) with increased levels of phosphorylated AMPK $[13,14]$, which has complex properties on cardiomyocyte functionality and ROS production [12]. In this context, we hypothesized that metformin played a direct protective role against I/R injury in diabetic hearts and we 
tested this hypothesis in an in vitro study using H9C2 rat cardiomyoblasts exposed to $\mathrm{H} / \mathrm{R}$ injury under a simulated hyperglycemic (HG) condition with or without coincubation with various concentrations of metformin. We further investigated the potential cellular and molecular mechanisms underlying metformin-induced cytoprotection against HG and/or H/R injury, particularly those related to the AMPK and JNK related kinase signaling pathways. Cellular ROS generation and proinflammatory cytokines were also investigated.

\section{Methods}

2.1. Cell Culture and Treatment Protocol. H9C2 rat cardiomyoblast cell line was purchased from the American Type Culture Collection (ATCC) and cultured in mixed growth medium (Dulbecco's modified Eagle's medium (DMEM) (Hyclone)) supplemented with 10\% heat-inactivated FBS (Hyclone). Cells were kept in an incubator in an atmosphere of $5 \% \mathrm{CO}_{2}$ and $95 \%$ air at $37^{\circ} \mathrm{C}$ and passaged at $1: 3$ ratio when they reached $80 \%$ confluence. For the H/R experimental groups, cells were firstly maintained at $37^{\circ} \mathrm{C}$ under hypoxic atmosphere of $95 \% \mathrm{~N}_{2}$ and $5 \% \mathrm{CO}_{2}$ for 3 hours, and then cells were given fresh medium with serum and maintained in normoxic conditions (i.e., reoxygenation) for the next 3 hours. The oxygen content $\left(\sim 1 \% \mathrm{O}_{2}\right)$ inside the incubator was continuously monitored to maintain a stable level of hypoxia. In the HG (33 mM)- and metformin $(0,1,5$, and $10 \mathrm{mM})$ treated groups, the cells were pretreated with glucose for 48 hours with or without an inhibitor of AMPK (compound C (Tocris)) or an activator of JNK (anisomycin (ANISO, Sigma-Aldrich)) followed by preincubation for $30 \mathrm{~min}$ before addition of metformin, which was added $60 \mathrm{~min}$ prior to $\mathrm{H} / \mathrm{R}$ until the end of the experiment protocol. JNK inhibitor (SP600125, Sigma-Aldrich) was added $30 \mathrm{~min}$ ahead of H/R. The cells in the control group were treated with the same procedure under normal culture conditions.

2.2. Cell Viability. After exposure to the abovementioned HG and/or H/R treatments with or without various concentrations of metformin $(0,1,5$, and $10 \mathrm{mM})$ and other kinase activators/inhibitors, cell proliferation in the H9C2 cells was assessed using Cell Counting Kit-8 (CCK-8, Sigma-Aldrich) according to the manufacturer's instruction. Briefly, cells were seeded on 96-well plates at a density of $2 \times 10^{3}$ per well in $100 \mu \mathrm{L}$ of complete medium. After being treated with the corresponding $\operatorname{drug}(\mathrm{s})$ and $\mathrm{H} / \mathrm{R}$ as described above, the cells were further incubated with $10 \mu \mathrm{L}$ of the CCK-8 reagent for 0.5 to 4 hours. The absorbance at $450 \mathrm{~nm}$ wavelength was measured with a microplate reader (BIO-TEX ELx800).

2.3. Measurement of Mitochondrial Membrane Potential. As a mitochondria specific membrane potential-sensitive fluorescent probe, JC-1 (Keygen) accumulates in the mitochondrial matrix when mitochondrial membrane potential is high, forming J-aggregates which emit bright yellow-red fluorescence. In contrast, JC-1 presents itself as a green fluorescent monomer with low levels of aggregation when mitochondrial membrane potential is low. During this assay, cells were seeded on 6-well plates and, following the assigned drug and/or H/R treatments, the cells were loaded with JC-1 for $20 \mathrm{~min}$ at $37^{\circ} \mathrm{C}$ and then harvested to detect fluorescence with a flow cytometer (BD Biosciences).

\subsection{Measurement of Activities of Mitochondrial Complex I and} Complex III. Mitochondrial complex activities were measured by a commercial assay kit for mitochondrial complex activity (Beyotime Institute of Biotechnology, China) following the manufacturer's instruction. Briefly, NADH-cytochrome c reductase activity (complexes I-III) was measured at $550 \mathrm{~nm}$ in a reaction medium containing (mM) 100 phosphate buffer ( $\mathrm{pH} 7.4$ ), 0.2 NADH, 0.1 cytochrome $\mathrm{c}$, and $0.5 \mathrm{KCN}$ at $30^{\circ} \mathrm{C}$. Enzyme activity was expressed in nmol cytochrome $\mathrm{c}$ reduced per minute per $\mathrm{mg}$ of protein. The activity was measured by the absorbance at $340 \mathrm{~nm}$ wavelength with a microplate reader (BIO-TEX ELx800) for $3 \mathrm{~min}$.

2.5. Flow Cytometric Evaluation of Intracellular ROS. The ROS generation was monitored by flow cytometry using peroxide-sensitive fluorescent probe 2,7-dichlorofluorescein diacetate (DCFH-DA) as previously described [15]. The cells were loaded with $10 \mathrm{mM}$ DCFH-DA in serum-free medium at $37^{\circ} \mathrm{C}$ for $30 \mathrm{mins}$, then washed twice with PBS, and then monitored with a flow cytometer (BD Biosciences) at an excitation wavelength of $488 \mathrm{~nm}$ and an emission wavelength of $525 \mathrm{~nm}$. ROS was determined by comparing the changes in fluorescence intensity to those of the control wells. The vertical coordinate represents the amount of cells, and the horizontal ordinate represents the mean fluorescence. The more the curve shifts to the right, the stronger the mean fluorescence is.

2.6. Real-Time PCR (Polymerase Chain Reaction) and $m R N A$ Levels of Cytokines. Total RNA from the cultured cells was extracted with TRIzol reagent (Invitrogen, Carlsbad, CA), followed by the synthesis of first-strand cDNA (Thermo). Real-time quantitative PCRs were performed using the SYBR-green I Core Kit (Thermo). PCR products were detected in the ABI PRISM 7700 sequence detection system (Applied Biosystems), and the results were analyzed using the $2^{-\Delta \Delta C T}$ method. The level of expression of mRNA was normalized to mRNA of GAPDH. Sequences of the primers are shown below:

$$
\begin{aligned}
& \text { TNF- } \alpha \text { : } \\
& \text { F5'-GGTCTGAGTACATCAACCTGGA-3', } \\
& \text { R5'-GGTCTGAGTACATCAACCTGGA-3'. } \\
& \text { IL-1 } \alpha \text { : } \\
& \text { F5'-AAGACAAGCCTGTGTTGCTGAAGG-3', } \\
& \text { R5' -TCCCAGAAGAAAATGAGGTCGGTC-3'. } \\
& \text { IL-6: } \\
& \text { F5'-TCAAGGGAAAAGAACCAGACA-3', } \\
& \text { R5' -TCAAGGGAAAAGAACCAGACA-3'. } \\
& \text { GAPDH: } \\
& \text { F5'-CTCTCTGCTCCTCCCTGTTC-3', } \\
& \text { R'5' }{ }^{\prime} \text {-GCCAAATCCGTTCACACCG- } 3^{\prime} .
\end{aligned}
$$


2.7. Western Blotting. Western blotting was performed as previously described [15]. Briefly, equal amount of protein extraction was loaded into an electrophoresis apparatus and, after the completion of electrophoresis, the blot was transferred onto a PVDF membrane followed by timed incubation with the selected primary and secondary antibodies. The scanned images of Western blot bands were analyzed using the Quantity One software (Bio-Rad). The levels of protein expression were quantified by densitometry and normalized to $\beta$-actin expression. Primary antibodies including phosphorylated and total AMPK (P-AMPK/TAMPK) and phosphorylated and total ACC (P-ACC/T-ACC) were purchased from Abcam, and phosphorylated and total JNK (P-JNK/T-JNK) antibodies were from Cell Signaling Technology.

2.8. Statistical Analyses. Each of the cellular and molecular biology assays was replicated with 3 independent experiments and the flow cytometry studies were repeated 6 times. All data were presented as mean \pm standard deviation (SD). Comparisons between groups were performed by one-way ANOVA with Student-Newman-Keuls post hoc analyses. The level for significant statistical differences was set at $P<0.05$.

\section{Results}

3.1. Metformin Protected against $H G$ and $H / R$ Induced Cardiac Cell Injury. To observe the effect of metformin on cell viability, H9C2 cells were exposed to HG and H/R treatments along with various concentrations of metformin. As evidenced by CCK-8 assay, the cell viability of HG or $H / R$ groups was significantly lower than those of control group and the cells exposed to $\mathrm{HG}+\mathrm{H} / \mathrm{R}$ had greater loss in cell viability as compared with $\mathrm{HG}$ and $\mathrm{H} / \mathrm{R}$ alone groups. Introducing low concentrations ( 1 or $5 \mathrm{mM}$ ) of metformin into the cell culture medium significantly increased the H9C2 cells viability while a higher concentration of metformin $(10 \mathrm{mM})$ aggravated the loss in cell viability induced by HG $+\mathrm{H} / \mathrm{R}$ treatment (Figure 1), indicating dose-dependence for metformin-induced protective effects, and only a moderate concentration of metformin is cytoprotective against HG + H/R injury.

3.2. Dependence of AMPK Activation in Protection of Metformin against $H / R$ Injury. It was reported that metformin protected cardiomyocytes from injury through activation of AMPK pathway $[13,14]$. To examine whether AMPK pathway inactivation was involved in $\mathrm{HG}$ and/or $\mathrm{H} / \mathrm{R}$ induced cardiac cell injury, we tested the phosphorylation state of AMPK and its downstream target ACC. Our results showed that the levels of P-AMPK were markedly lower and its inhibitory downstream target $\mathrm{P}-\mathrm{ACC}$ were higher in $\mathrm{HG}$ or $\mathrm{H} / \mathrm{R}$ groups than in control group (Figures 2(a) and 2(b)) and the cells in HG $+\mathrm{H} / \mathrm{R}$ group had the lowest level of P-AMPK (Figure 2(a)) and the resultant highest level of P-ACC (Figure 2(b)). These results suggested that AMPK pathway was inhibited during $\mathrm{H} / \mathrm{R}$ injury and this effect was exacerbated under HG + $\mathrm{H} / \mathrm{R}$ injury. Incubation with all three concentrations of metformin $(1,5$, and $10 \mathrm{mM})$ significantly increased P-AMPK

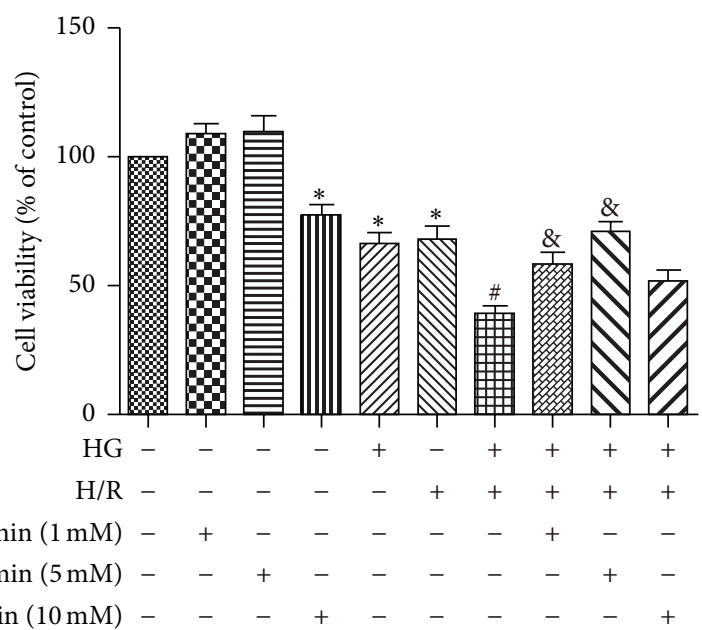

Figure 1: Dose-dependent effects of metformin on $\mathrm{HG}+\mathrm{H} / \mathrm{R}$ induced reduction in cell viability. $\mathrm{H} 9 \mathrm{C} 2$ cells were treated with different concentrations of metformin $(0,1,5$, and $10 \mathrm{mM})$. A "+" symbol indicates presence and a "-" symbol indicates absence of the relevant treatment condition, such as $H G(33 \mathrm{mM}), H / R$, and various concentrations of metformin. Cell viability of each group was estimated using the CCK- 8 assay. Data are shown as means \pm SD of 3 independent experiments. ${ }^{*} P<0.05$ versus Control; ${ }^{\#} P<0.05$ versus $\mathrm{HG}$ or $\mathrm{H} / \mathrm{R} ;{ }^{\circledR} \mathrm{P}<0.05$ versus metformin $+\mathrm{HG}+\mathrm{H} / \mathrm{R}$.

expression and the highest level of P-AMPK was found in the cytoprotective dose $(5 \mathrm{mM})$ of metformin (Figure 2(c)). Furthermore, the metformin-enhanced cell viability under HG $+\mathrm{H} / \mathrm{R}$ conditions was abolished by compound $\mathrm{C}$, an inhibitor of AMPK (Figure 2(d)), confirming that metformin protected against $\mathrm{H} / \mathrm{R}+\mathrm{HG}$ injury by activating AMPK pathway.

\subsection{Metformin Attenuates $H G+H / R$ Induced Reduction in} Mitochondrial Transmembrane Potential and Increased ROS Formation. Mitochondria act as a nexus for reperfusion injury pathways [16] and diffusion of mitochondrial transmembrane potential $(\Delta \psi m)$ indicates mitochondrial dysfunction [17]. In our present study, a mitochondrial membrane potential kit was used to explore the effect of metformin on mitochondrial function in cells exposed to HG and H/R. Metformin ameliorated depolarized $\Delta \psi m$ induced by HG $+\mathrm{H} / \mathrm{R}$ treatment, which was abolished by compound $\mathrm{C}$ evidenced from the data of JC-1 (Figure 3(a)).

$\mathrm{I} / \mathrm{R}$ injury impairs the mitochondrial respiratory chain, especially complexes I and III, and produces a large amount of ROS [18]. To investigate the effect of metformin on ROS levels in the cells exposed to HG and H/R, DCF-DA assay was performed. The levels of ROS in the $\mathrm{HG}+\mathrm{H} / \mathrm{R}$ groups treated with low dose metformin $(5 \mathrm{mM})$ were significantly lower than that of the HG $+\mathrm{H} / \mathrm{R}$ group (Figures 3(b)$3(c))$. On the other hand, metformin increased the activity of mitochondrial electron transport chain complexes I and III that were reduced by HG $+\mathrm{H} / \mathrm{R}$ (Figure 3(c)). Consistent with JC-1, the ROS reduction and mitochondrial electron transport chain complex activity increase by metformin treatment were reversed by compound C (Figures 3(b)-3(d)). 


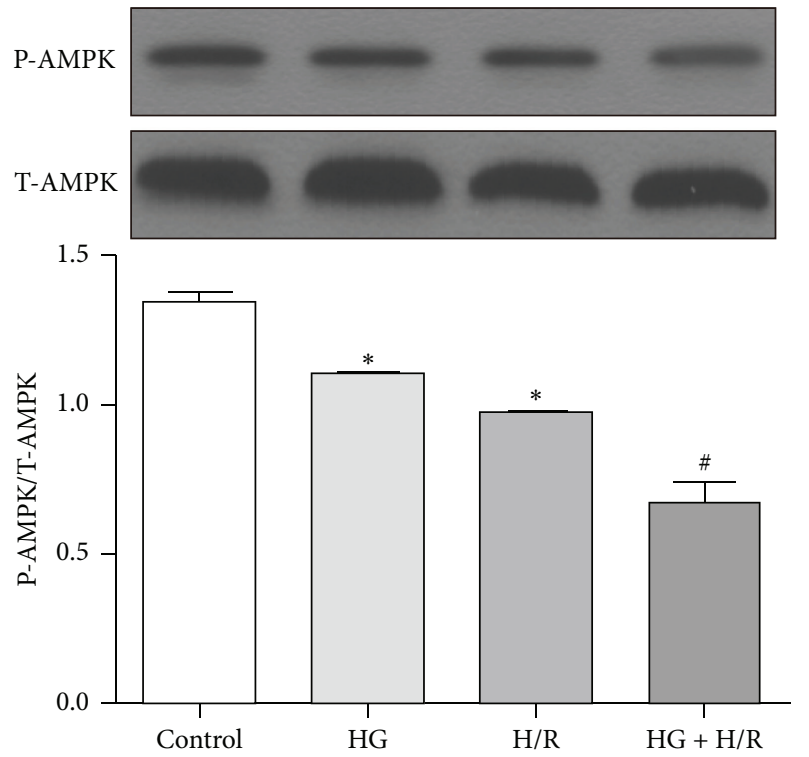

(a)

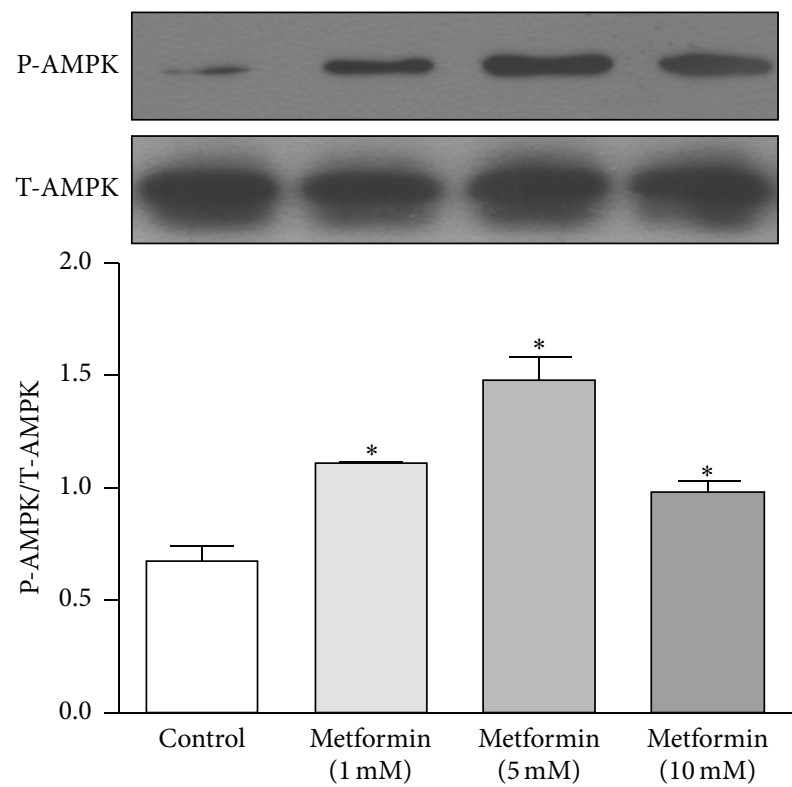

(c)

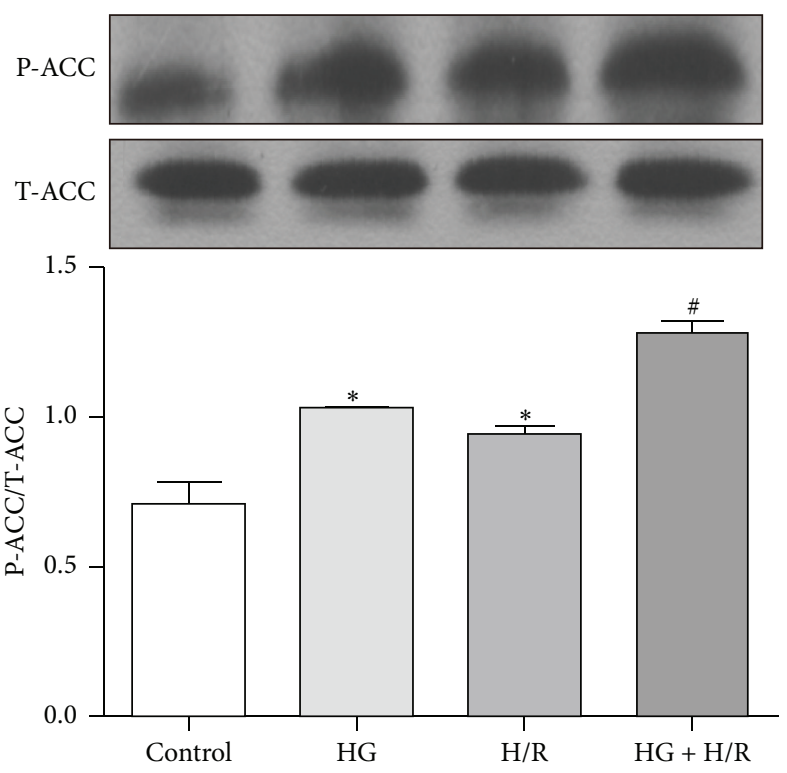

(b)

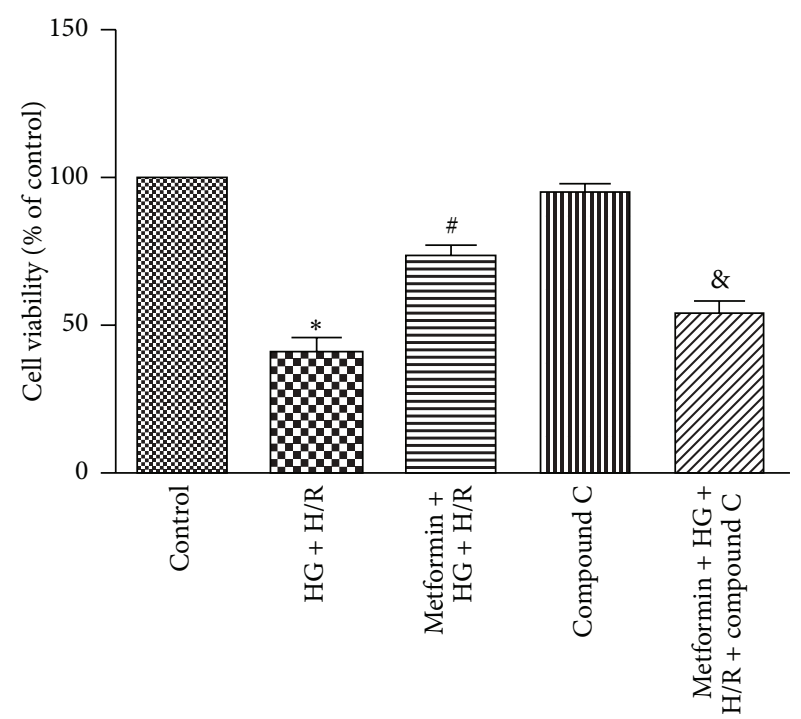

(d)

FIGURE 2: AMPK pathway was inhibited during H/R injury and exacerbated by HG and the cytoprotective effects of metformin were dependent on AMPK activation. H9C2 cells were treated with HG $(33 \mathrm{mM}), \mathrm{H} / \mathrm{R}$, and HG + H/R. The expression of P-AMPK, T-AMPK (a) and P-ACC, T-ACC (b) was measured by Western blots. (c) Expression of P-AMPK and T-AMPK in the H9C2 cells treated with different concentrations of metformin $(0,1,5$, and $10 \mathrm{mM})$ was measured by Western blots. (d) Cells were treated with HG + H/R, metformin $(5 \mathrm{mM})$ $+\mathrm{HG}+\mathrm{H} / \mathrm{R}$, compound $\mathrm{C}(1 \mu \mathrm{M})$, or metformin $+\mathrm{HG}+\mathrm{H} / \mathrm{R}+$ compound C. Cell viability of each group was established using the CCK-8 assay. ${ }^{*} P<0.05$ versus control; ${ }^{\#} P<0.05$ versus $\mathrm{HG}$ or $\mathrm{H} / \mathrm{R} ;{ }^{\circledR} \mathrm{P}<0.05$ versus metformin $+\mathrm{HG}+\mathrm{H} / \mathrm{R}$. Data are shown as means $\pm \mathrm{SD}$ of 3 independent experiments.

3.4. Metformin Inhibits Inflammatory Response to $H G$ and $H / R$ Injury. Exposure to $\mathrm{HG}+\mathrm{H} / \mathrm{R}$ injury induced an inflammatory response, characterized by increasing $\mathrm{mRNA}$ levels of IL- $1 \alpha$, IL- 6 , and TNF- $\alpha$ compared with that of control groups (Figure 4) and administration of metformin (5 mM) reduced these changes. In addition, inhibition of AMPK by compound $\mathrm{C}$ reversed this trend, implying that the protective effect of metformin against $\mathrm{HG}+\mathrm{H} / \mathrm{R}$ induced inflammation was mediated by activation of AMPK signaling pathway.
3.5. Metformin Protects against $H G+H / R$ Injury by Inhibiting Phosphorylation of JNK, Which Is a Downstream Target of AMPK. Expression of P-JNK and T-JNK in each of the treatment conditions was measured by Western blots (Figure 5(a)). The results showed that increased protein level of P-JNK during $\mathrm{HG}+\mathrm{H} / \mathrm{R}$ treatment was also decreased by metformin $(5 \mathrm{mM})$ and inhibition of AMPK by compound $\mathrm{C}$ reversed these changes in P-JNK expression. In addition, the protective effect of metformin was abolished by cotreatment 


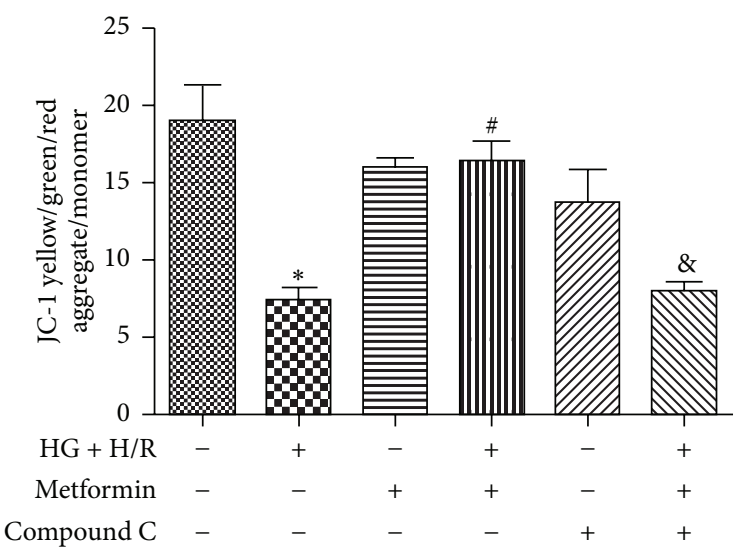

(a)
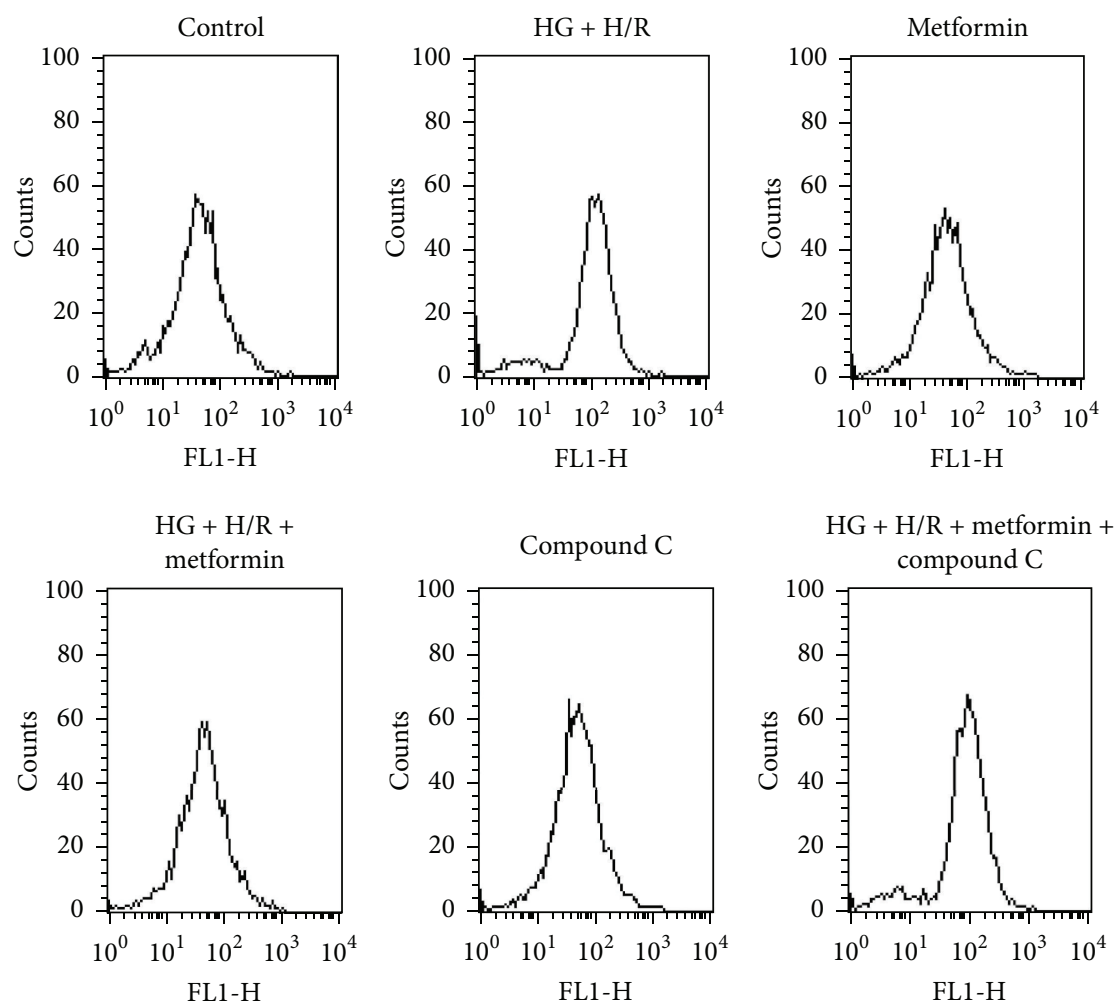

(b)

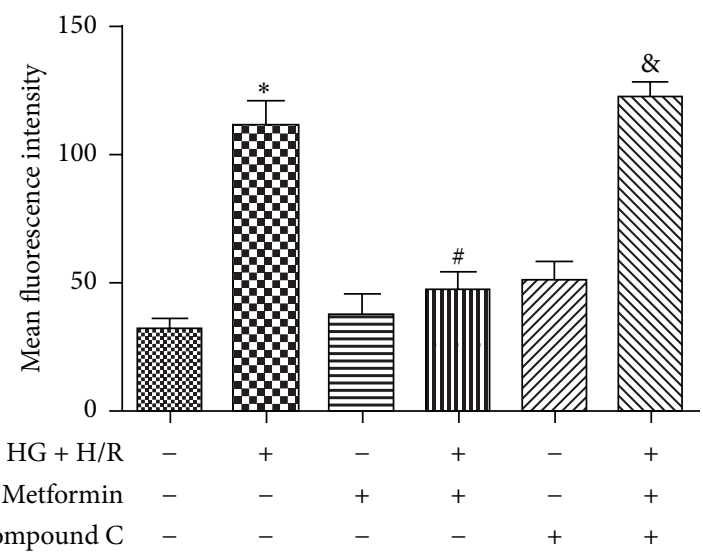

(c)

Figure 3: Continued. 

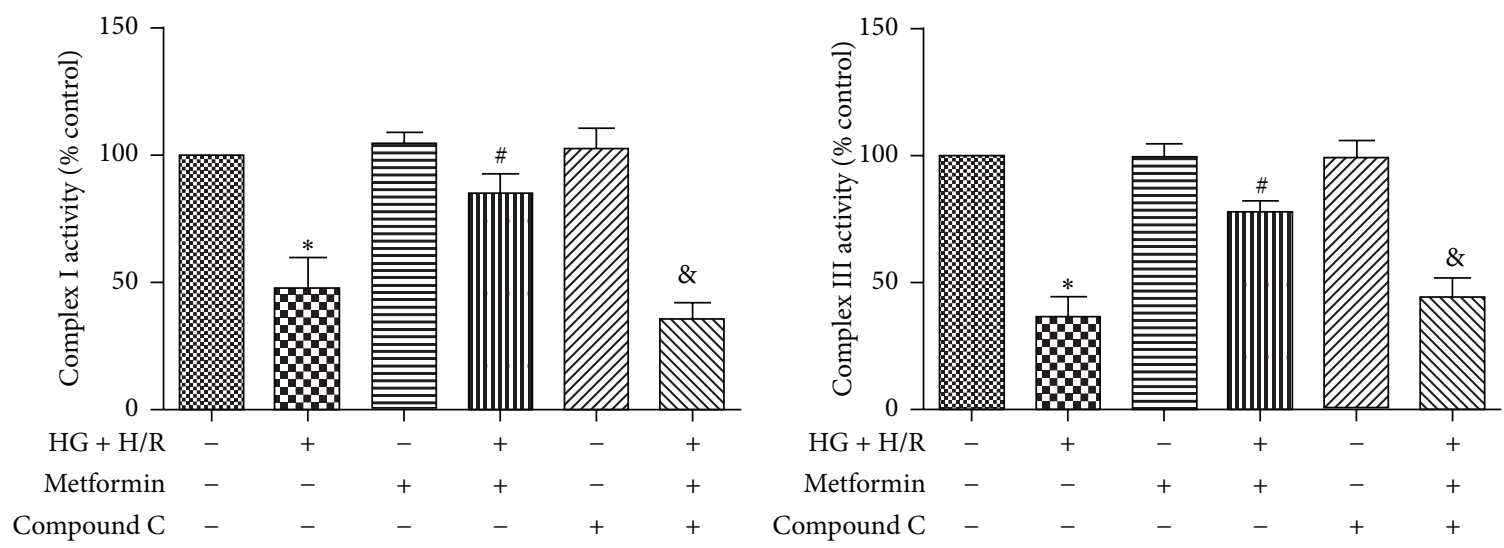

(d)

FIGURE 3: Metformin attenuated HG $+\mathrm{H} / \mathrm{R}$ induced decrease in cell viability by preservation of mitochondrial membrane integrity and mitigating oxidative stress. H9C2 cells were treated with $\mathrm{HG}+\mathrm{H} / \mathrm{R}$, metformin $(5 \mathrm{mM})$, metformin $+\mathrm{HG}+\mathrm{H} / \mathrm{R}$, compound C $(1 \mu \mathrm{M})$, and metformin $+\mathrm{HG}+\mathrm{H} / \mathrm{R}+$ compound C. (a) Mitochondrial membrane potential evidenced by JC-1 staining. (b) ROS generation was measured by the DCF fluorescence intensity. (c) Bars represent quantified ROS generation. (d) Mitochondrial electron transport chain complex I and complex III activities were measured with commercial kits. ${ }^{*} P<0.05$ versus control; ${ }^{*} P<0.05$ versus $\mathrm{HG}+\mathrm{H} / \mathrm{R} ;{ }^{\circledR} P<0.05$ versus metformin $+\mathrm{HG}+\mathrm{H} / \mathrm{R}$. Data in the bar graphs are calculated as means $\pm \mathrm{SD}$ of 3 independent experiments.

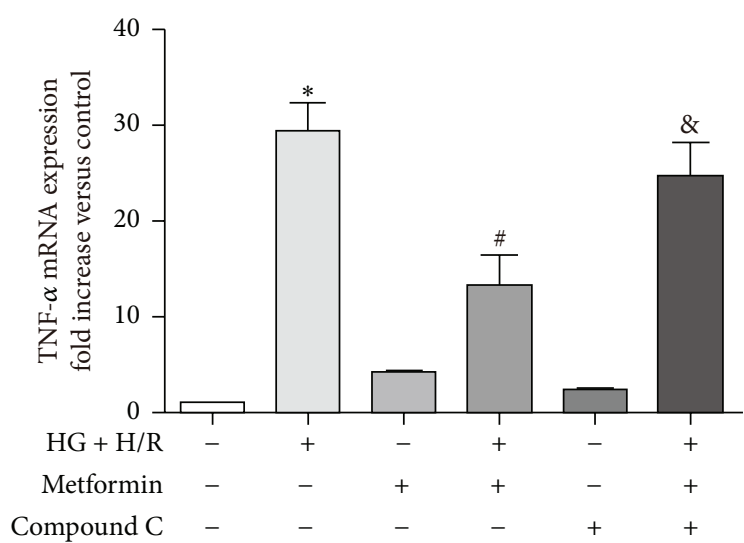

(a)

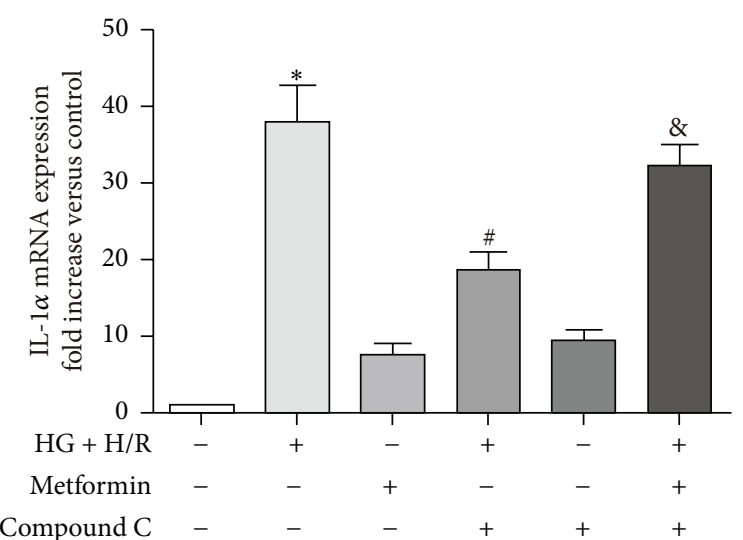

(b)

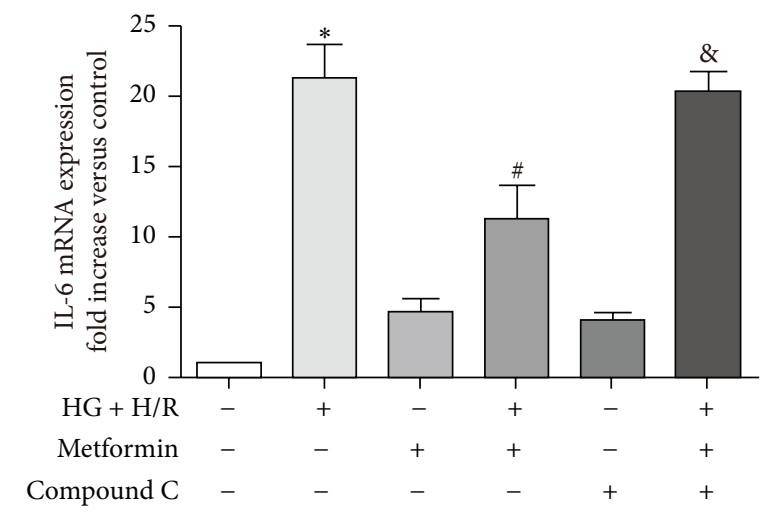

(c)

FIGURE 4: Metformin attenuated HG and H/R injury by inhibiting proinflammatory cytokine expression in cardiac cells. H9C2 cells were treated as described in Figure 3. (a)-(c) Expressions of TNF- $\alpha$, IL- $1 \alpha$, and IL-6 mRNA level were measured by real-time PCR. GAPDH was used as the housekeeping gene. Data are shown as means $\pm \mathrm{SD}$ of 3 independent experiments. ${ }^{*} P<0.05$ versus control; ${ }^{\#} P<0.05$ versus $\mathrm{HG}$ $+\mathrm{H} / \mathrm{R} ;{ }^{\&} \mathrm{P}<0.05$ versus metformin $+\mathrm{HG}+\mathrm{H} / \mathrm{R}$. 


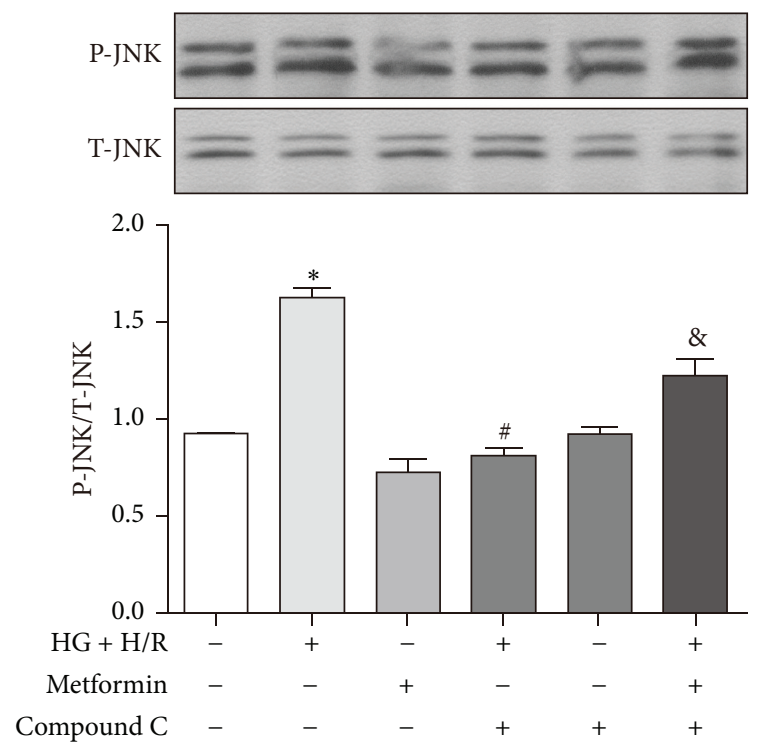

(a)

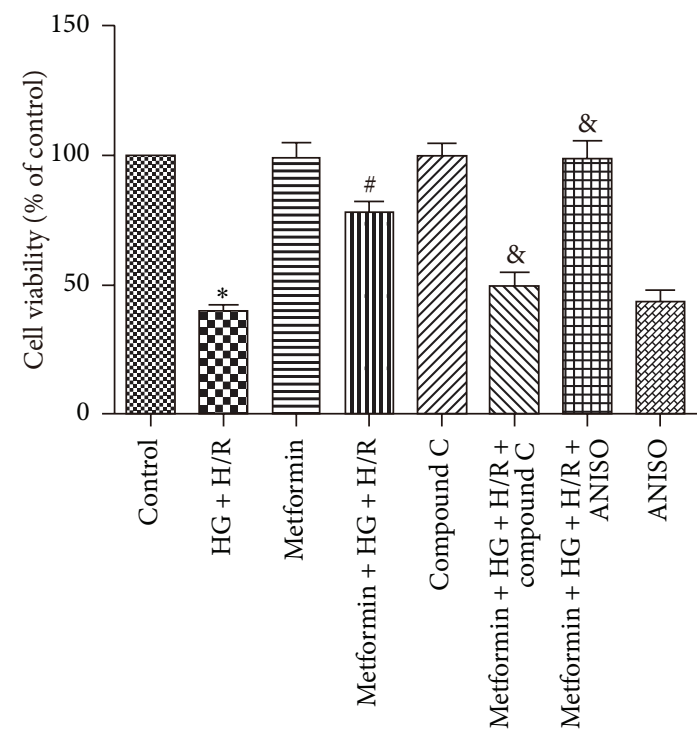

(b)

FIGURE 5: Metformin protects against HG + H/R induced cardiac cell injury by inhibiting JNK signaling pathway, which acts downstream of AMPK. H9C2 cells were treated with $\mathrm{HG}+\mathrm{H} / \mathrm{R}$, metformin $(5 \mathrm{mM})$, metformin $+\mathrm{HG}+\mathrm{H} / \mathrm{R}$, compound $\mathrm{C}(1 \mu \mathrm{M})$, metformin $+\mathrm{HG}+$ $\mathrm{H} / \mathrm{R}+$ compound C, ANISO $(10 \mu \mathrm{M})$, and metformin $+\mathrm{HG}+\mathrm{H} / \mathrm{R}+$ ANISO. (a) Expression of P-JNK and T-JNK in each of the treatment conditions was measured by Western blots. (b) Effect of a putative JNK activator ANISO on cell viability was determined using the CCK-8 kit. Data are shown as means $\pm \mathrm{SD}$ of 3 independent experiments. ${ }^{*} P<0.05$ versus control; ${ }^{\#} P<0.05$ versus $\mathrm{HG}+\mathrm{H} / \mathrm{R} ;{ }^{8} P<0.05$ versus metformin $+\mathrm{HG}+\mathrm{H} / \mathrm{R}$.

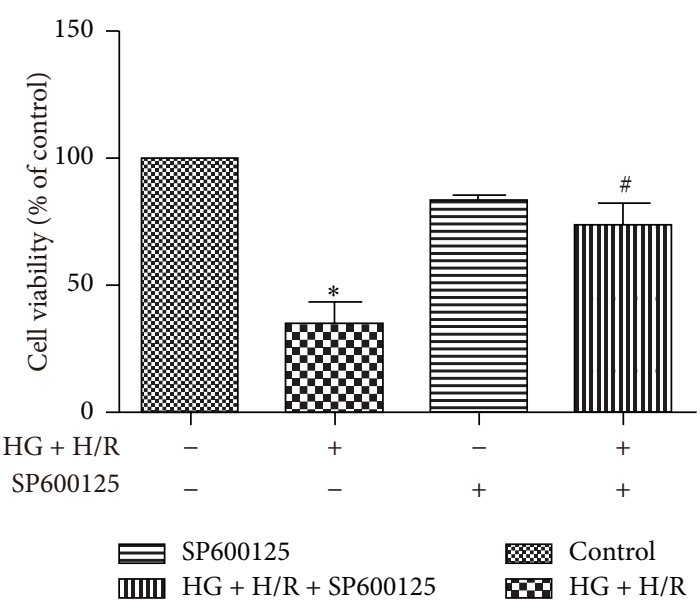

(a)

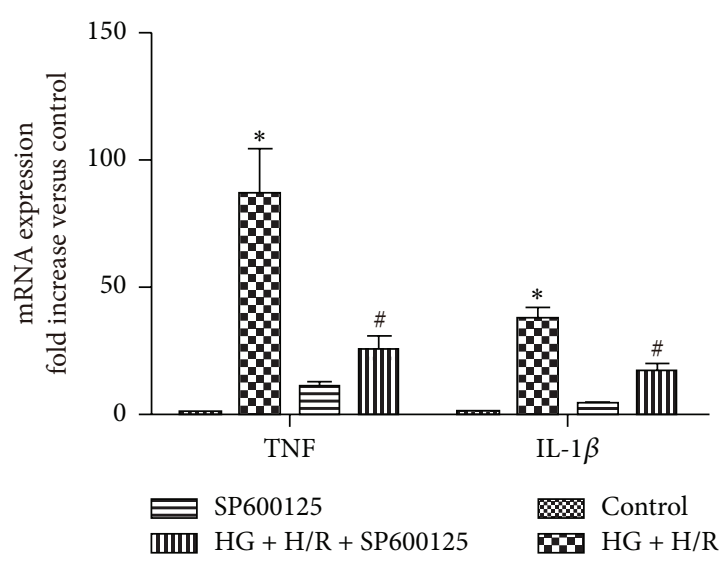

(b)

FIGURE 6: Protection of JNK inhibitor SP600125 against HG + H/R induced loss of cell viability and proinflammatory cytokines release. (a) Protective effects of SP600125 on HG + H/R induced loss of cell viability. (b) Effect of SP600125 on HG + H/R induced proinflammatory cytokine expression. Data are shown as mean $\pm \mathrm{SD}$ of 3 independent experiments. ${ }^{*} P<0.05$ versus control; ${ }^{\#} P<0.05$ versus $\mathrm{HG}+\mathrm{H} / \mathrm{R}$.

with a putative JNK activator, ANISO (Figure 5(b)), indicating that the inhibition of JNK is required for the metformininduced cytoprotection.

3.6. JNK Inhibition Reduced HG and H/R Injury. To further demonstrate that JNK signal pathway plays an essential role in $\mathrm{HG}$ and $\mathrm{H} / \mathrm{R}$ induced cardiac injury, we performed additional experiments and found that a JNK inhibitor (SP600125) prevented the $\mathrm{HG}+\mathrm{H} / \mathrm{R}$ induced loss of cell viability and suppressed the inflammation cytokines synthesis under HG $+\mathrm{H} / \mathrm{R}$ (Figure 6).

\section{Discussion}

The main finding of our study was that metformin protected cardiac cells against $\mathrm{HG}+\mathrm{H} / \mathrm{R}$ injury by a mechanism involving P-AMPK, ROS, and JNK signaling pathways. Patients with diabetes mellitus are at higher risk of cardiovascular events compared with nondiabetic individuals as evidenced by numerous clinical studies $[19,20]$. For instance, diabetic patients are more vulnerable to ischemic heart diseases $[3,4]$. Unfortunately, many therapeutic strategies that have been shown to be effective in the protection of nondiabetic hearts 
against I/R injury often lose their effectiveness in diabetic states [21, 22]. Although metformin has been reported to protect diabetic mouse heart against I/R injury [23, 24], its underlying mechanisms remain largely unknown. Our current study showed that metformin at a low concentration $(5 \mathrm{mM})$ reduced the cardiac cell death caused by $\mathrm{HG}+\mathrm{H} / \mathrm{R}$ injury and metformin alone had no effect on cell viability. However, a higher concentration of metformin $(10 \mathrm{mM})$ dramatically decreased cell viability. This cytotoxic effect of higher dose of metformin may result from the excessive enhancement of AMPK activity that led to suppression of platelet-derived growth factor receptor (PDGFR) as previously reported in H9C2 cells [25].

ROS plays a key role in $\mathrm{HG}+\mathrm{H} / \mathrm{R}$ induced cardiac injury and most of the ROS are generated within the impaired mitochondria. Metformin has been shown to reduce HGinduced ROS generation and oxidative stress in endothelial cells $[26,27]$. Therefore, we investigated whether metformin had a suppressive effect against $\mathrm{HG}+\mathrm{H} / \mathrm{R}$ induced ROS overgeneration in cardiomyocytes. We first found that mitochondrial transmembrane potential $(\Delta \psi m)$ was diffused after $\mathrm{HG}+\mathrm{H} / \mathrm{R}$ treatment indicating mitochondrial dysfunction and this effect was reversed by metformin $(5 \mathrm{mM})$ coincubation (Figure 3(a)). In line with the result of mitochondrial function, metformin administration reduced the increase in ROS generation stimulated by $\mathrm{HG}+\mathrm{H} / \mathrm{R}$ (Figures 3(b)3(c)). We subsequently examined whether the ROS was produced at the site of mitochondrion. As expected, the activity of mitochondrial electron transport chain complex $\mathrm{I}$ and complex III was reduced by $\mathrm{HG}+\mathrm{H} / \mathrm{R}$ injury and metformin attenuated this effect (Figure 3(d)). Overall, our data suggested that metformin protected heart cells against $\mathrm{HG}+\mathrm{H} / \mathrm{R}$ injury by inhibiting overproduction of ROS derived most likely from mitochondria.

Oxidative stress links multiple risk factors to disease and one of the main possible underlying mechanisms is that overproduction of ROS stimulates inflammatory response [28]. We found here that $\mathrm{HG}+\mathrm{H} / \mathrm{R}$ induced cardiac cell injury was associated with activation of inflammatory response as evidenced by the significant increase in mRNA of proinflammatory cytokines (TNF- $\alpha$, IL- $1 \alpha$, and IL- 6 ) and metformin mitigated these increases (Figures 4(a)-4(c)). Previous studies reported that exogenous ROS could stimulate JNK in H9C2 cells $[29,30]$. Consistent with these previous studies, our present research also demonstrated that $\mathrm{HG}+\mathrm{H} / \mathrm{R}$ injury induced cardiac ROS overproduction was associated with an increase in P-JNK expression, which was inhibited by metformin administration (Figures 6(a)-6(b)).

Previous rat study reported that metformin activated AMPK pathway and protected against myocardial I/R injury [31]. To investigate whether metformin protected $\mathrm{H} 9 \mathrm{C} 2$ cells against $\mathrm{HG}+\mathrm{H} / \mathrm{R}$ injury also through activation of AMPK, we used AMPK inhibitor compound $C$ and found that the protective effect of metformin against $\mathrm{HG}+\mathrm{H} / \mathrm{R}$ injury was abolished by compound $\mathrm{C}$ treatment (Figure 5(b)). We further uncovered that the AMPK activation and cytoprotective effect of metformin were interconnected with inhibition on P-JNK and compound C restored the metformin-induced suppression of JNK, indicating that AMPK activation was an upstream event of JNK inhibition caused by metformin (Figures 6(a)-6(b)). Furthermore, the JNK activator anisomycin (ANISO) antagonized the inhibitory effects of metformin on $\mathrm{HG}+\mathrm{H} / \mathrm{R}$ induced JNK activation and also blocked cytoprotection of metformin (Figure 5(b)). Finally, our results provided additional evidence for a detrimental role played by JNK in HG + H/R injury, because a JNK inhibitor (SP600125) reduced the cell injury (Figure 6(a)) and proinflammatory cytokine response (Figures 6(a)-6(b)) caused by HG + H/R.

\section{Conclusion}

The current study has revealed for the first time that the in vitro direct treatment of metformin $(5 \mathrm{mM})$ in $\mathrm{H} 9 \mathrm{C} 2$ cardiomyoblasts attenuated $\mathrm{HG}$ and $\mathrm{H} / \mathrm{R}$ induced cell injury, mitochondrial dysfunction, ROS overgeneration, and inflammatory response through an AMPK/JNK-dependent signaling pathway. These results from cultured cardiac cells may also implicate an important mechanism by which metformin antagonizes myocardial I/R injury in vivo. Future studies are needed to further validate the role of this signaling mechanism in mediating metformin-induced cardioprotection in various animal species including human.

\section{Competing Interests}

The authors declare that there are no competing interests regarding the publication of this paper.

\section{Acknowledgments}

This study was supported by the Science Foundation of the Central Hospital of Wuhan, Hubei, China (YQ15B03).

\section{References}

[1] D. M. Nathan, "Long-term complications of diabetes mellitus," The New England Journal of Medicine, vol. 328, no. 23, pp. 16761685, 1993.

[2] G. Zuanetti, R. Latini, A. P. Maggioni, L. Santoro, and M. G. Franzosi, "Influence of diabetes on mortality in acute myocardial infarction: data from the GISSI-2 study," Journal of the American College of Cardiology, vol. 22, no. 7, pp. 1788-1794, 1993.

[3] A. Norhammar, J. Lindbäck, L. Rydén, L. Wallentin, and U. Stenestrand, "Improved but still high short- and long-term mortality rates after myocardial infarction in patients with diabetes mellitus: a time-trend report from the Swedish Register of Information and Knowledge about Swedish Heart Intensive Care Admission," Heart, vol. 93, no. 12, pp. 1577-1583, 2007.

[4] D. Feuvray, J. A. Idell-Wenger, and J. R. Neely, "Effects of ischemia on rat myocardial function and metabolism in diabetes," Circulation Research, vol. 44, no. 3, pp. 322-329, 1979.

[5] V. Sivaraman and D. M. Yellon, "Pharmacologic therapy that simulates conditioning for cardiac ischemic/reperfusion injury," Journal of Cardiovascular Pharmacology and Therapeutics, vol. 19, no. 1, pp. 83-96, 2014.

[6] B. Viollet, B. Guigas, N. Sanz Garcia, J. Leclerc, M. Foretz, and F. Andreelli, "Cellular and molecular mechanisms of metformin: an overview," Clinical Science, vol. 122, no. 6, pp. 253-270, 2012. 
[7] J. A. Johnson, S. H. Simpson, E. L. Toth, and S. R. Majumdar, "Reduced cardiovascular morbidity and mortality associated with metformin use in subjects with Type 2 diabetes," Diabetic Medicine, vol. 22, no. 4, pp. 497-502, 2005.

[8] J. A. Johnson, S. R. Majumdar, S. H. Simpson, and E. L. Toth, "Decreased mortality associated with the use of metformin compared with sulfonylurea monotherapy in type 2 diabetes," Diabetes Care, vol. 25, no. 12, pp. 2244-2248, 2002.

[9] S. Verma, S. Bhanot, and J. H. McNeill, "Decreased vascular reactivity in metformin-treated fructose-hypertensive rats," Metabolism: Clinical and Experimental, vol. 45, no. 9, pp. 10531055, 1996.

[10] R. C. Bhalla, K. F. Toth, E. Tan, R. A. Bhatty, E. Mathias, and R. V. Sharma, "Vascular effects of metformin. Possible mechanisms for its antihypertensive action in the spontaneously hypertensive rat," American Journal of Hypertension, vol. 9, no. 6, pp. 570576, 1996.

[11] C.-H. Yeh, J.-H. S. Pang, Y.-C. Wu, Y.-C. Wang, J.-J. Chu, and P. J. Lin, "Differential-display polymerase chain reaction identifies nicotinamide adenine dinucleotide-ubiquinone oxidoreductase as an ischemia/reperfusion-regulated gene in cardiomyocytes," Chest, vol. 125, no. 1, pp. 228-235, 2004.

[12] K. Li, Y.-C. Cui, H. Zhang et al., "Glutamine reduces the apoptosis of H9C2 cells treated with high-glucose and reperfusion through an oxidation-related mechanism," PLoS ONE, vol. 10, no. 7, Article ID e0132402, 2015.

[13] G. Zhou, R. Myers, Y. Li et al., "Role of AMP-activated protein kinase in mechanism of metformin action," The Journal of Clinical Investigation, vol. 108, no. 8, pp. 1167-1174, 2001.

[14] L. G. D. Fryer, A. Parbu-Patel, and D. Carling, "The anti-diabetic drugs rosiglitazone and metformin stimulate AMP-activated protein kinase through distinct signaling pathways," The Journal of Biological Chemistry, vol. 277, no. 28, pp. 25226-25232, 2002.

[15] R. Dong, X. Xu, G. Li et al., "Bradykinin inhibits oxidative stress-induced cardiomyocytes senescence via regulating redox state," PLoS ONE, vol. 8, no. 10, Article ID e77034, 2013.

[16] K. D. Patil, H. R. Halperin, and L. B. Becker, "Cardiac arrest: resuscitation and reperfusion," Circulation Research, vol. 116, no. 12, pp. 2041-2049, 2015.

[17] R. J. Mailloux, S. L. McBride, and M.-E. Harper, "Unearthing the secrets of mitochondrial ROS and glutathione in bioenergetics," Trends in Biochemical Sciences, vol. 38, no. 12, pp. 592-602, 2013.

[18] H. Cao, Y. Wang, Q. Wang et al., "Taxol prevents myocardial ischemia-reperfusion injury by inducing JNK-mediated HO-1 expression," Pharmaceutical Biology, vol. 54, no. 3, pp. 555-560, 2016.

[19] J. Ali Raza and A. Movahed, "Current concepts of cardiovascular diseases in diabetes mellitus," International Journal of Cardiology, vol. 89, no. 2-3, pp. 123-134, 2003.

[20] G. L. Min, H. J. Myung, Y. Ahn et al., "Comparison of clinical outcomes following acute myocardial infarctions in hypertensive patients with or without diabetes," Korean Circulation Journal, vol. 39, no. 6, pp. 243-250, 2009.

[21] M. Ishihara, I. Inoue, T. Kawagoe et al., "Diabetes mellitus prevents ischemic preconditioning in patients with a first acute anterior wall myocardial infarction," Journal of the American College of Cardiology, vol. 38, no. 4, pp. 1007-1011, 2001.

[22] S. L. Woodfield, C. F. Lundergan, J. S. Reiner et al., "Angiographic findings and outcome in diabetic patients treated with thrombolytic therapy for acute myocardial infarction: the GUSTO-I experience," Journal of the American College of Cardiology, vol. 28, no. 7, pp. 1661-1669, 1996.
[23] F. Abbasi, J. W. Chu, T. McLaughlin, C. Lamendola, E. T. Leary, and G. M. Reaven, "Effect of metformin treatment on multiple cardiovascular disease risk factors in patients with type 2 diabetes mellitus," Metabolism: Clinical and Experimental, vol. 53, no. 2, pp. 159-164, 2004.

[24] S. Verma and J. H. McNeill, "Metformin improves cardiac function in isolated streptozotocin-diabetic rat hearts," American Journal of Physiology-Heart and Circulatory Physiology, vol. 266, no. 2, pp. H714-H719, 1994.

[25] L. C. Kobashigawa, Y. C. Xu, J. F. Padbury, Y.-T. Tseng, and N. Yano, "Metformin protects cardiomyocyte from doxorubicin induced cytotoxicity through an AMP-activated protein kinase dependent signaling pathway: an in vitro study," PLOS ONE, vol. 9, no. 8, Article ID e104888, 2014.

[26] A. Gallo, G. Ceolotto, P. Pinton et al., "Metformin prevents glucose-induced protein kinase C- $\beta 2$ activation in human umbilical vein endothelial cells through an antioxidant mechanism," Diabetes, vol. 54, no. 4, pp. 1123-1131, 2005.

[27] D. Kukidome, T. Nishikawa, K. Sonoda et al., "Activation of AMP-activated protein kinase reduces hyperglycemia-induced mitochondrial reactive oxygen species production and promotes mitochondrial biogenesis in human umbilical vein endothelial cells," Diabetes, vol. 55, no. 1, pp. 120-127, 2006.

[28] H. Ebrahimi, R. Badalzadeh, M. Mohammadi, and B. Yousefi, "Diosgenin attenuates inflammatory response induced by myocardial reperfusion injury: role of mitochondrial ATPsensitive potassium channels," Journal of Physiology and Biochemistry, vol. 70, no. 2, pp. 425-432, 2014.

[29] I.-K. S. Aggeli, I. Beis, and C. Gaitanaki, "ERKs and JNKs mediate hydrogen peroxide-induced Egr-1 expression and nuclear accumulation in H9c2 cells," Physiological Research, vol. 59, no. 3, pp. 443-454, 2010.

[30] Y. Mo, R. Wan, L. Feng, S. Chien, D. J. Tollerud, and Q. Zhang, "Combination effects of cigarette smoke extract and ambient ultrafine particles on endothelial cells," Toxicology in Vitro, vol. 26, no. 2, pp. 295-303, 2012.

[31] M. A. Paiva, L. M. Gonçalves, L. A. Providência, S. M. Davidson, D. M. Yellon, and M. M. Mocanu, "Transitory activation of AMPK at reperfusion protects the ischaemic-reperfused rat myocardium against infarction," Cardiovascular Drugs and Therapy, vol. 24, no. 1, pp. 25-32, 2010. 


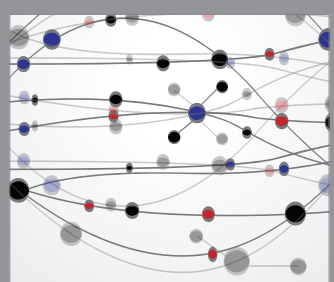

The Scientific World Journal
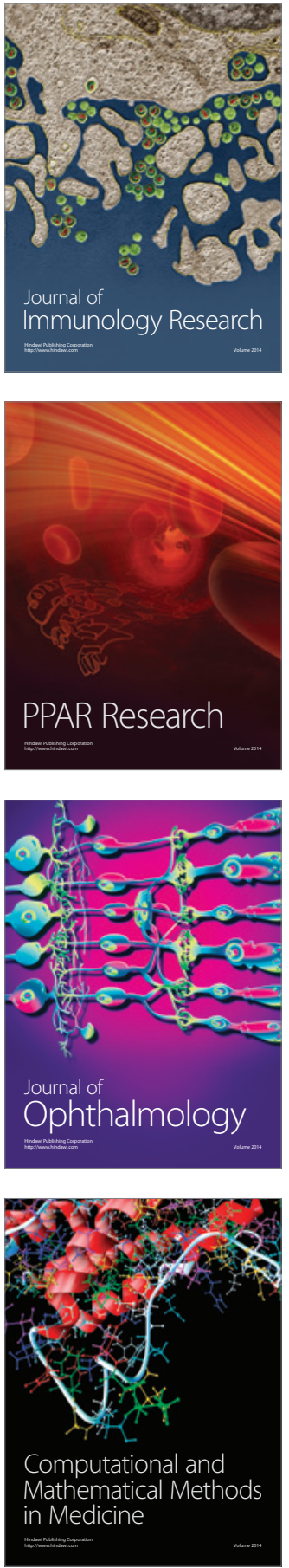

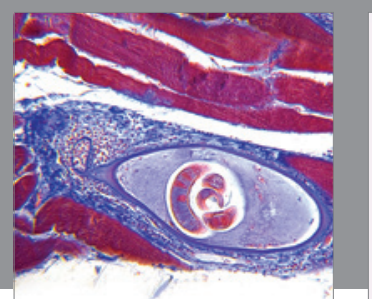

Gastroenterology Research and Practice

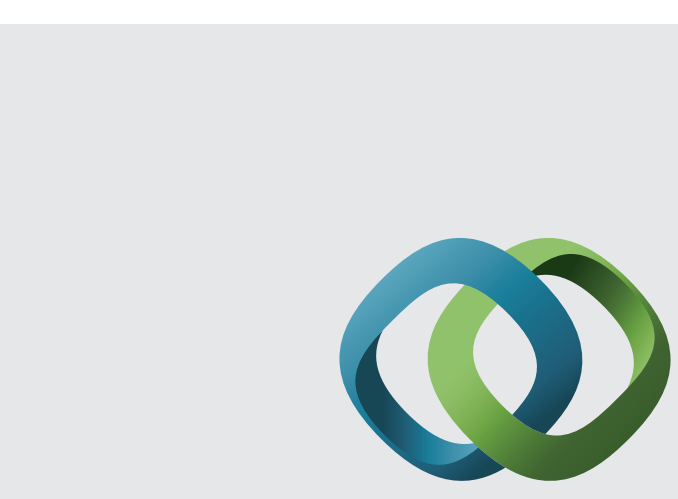

\section{Hindawi}

Submit your manuscripts at

http://www.hindawi.com
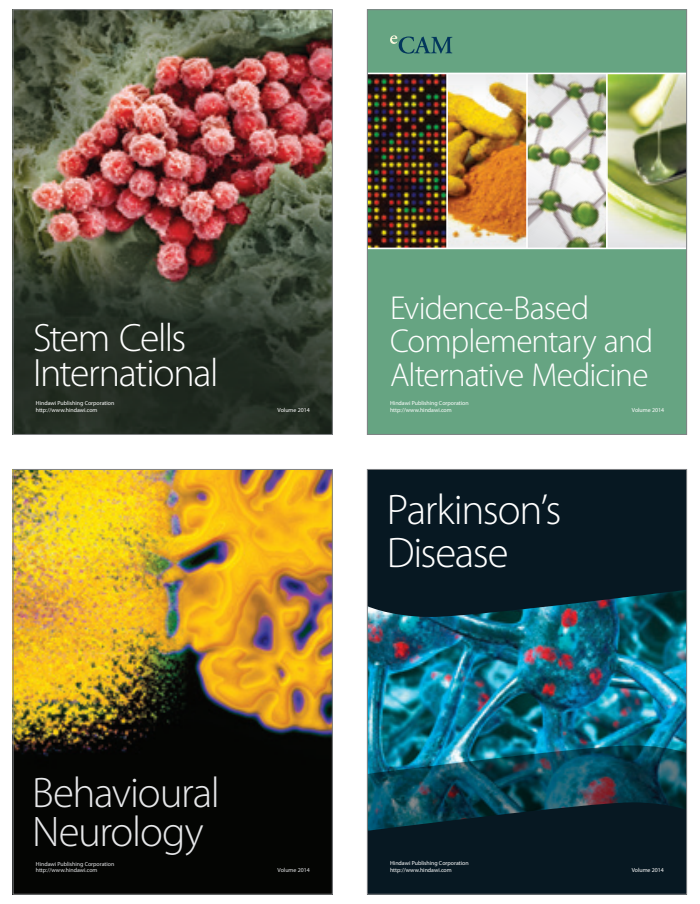
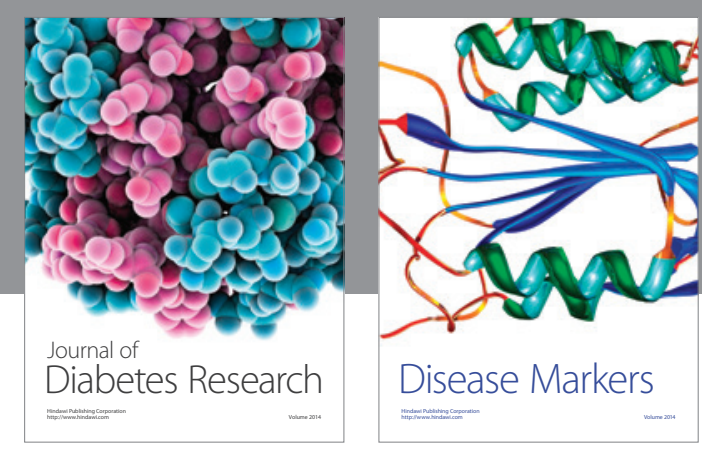

Disease Markers
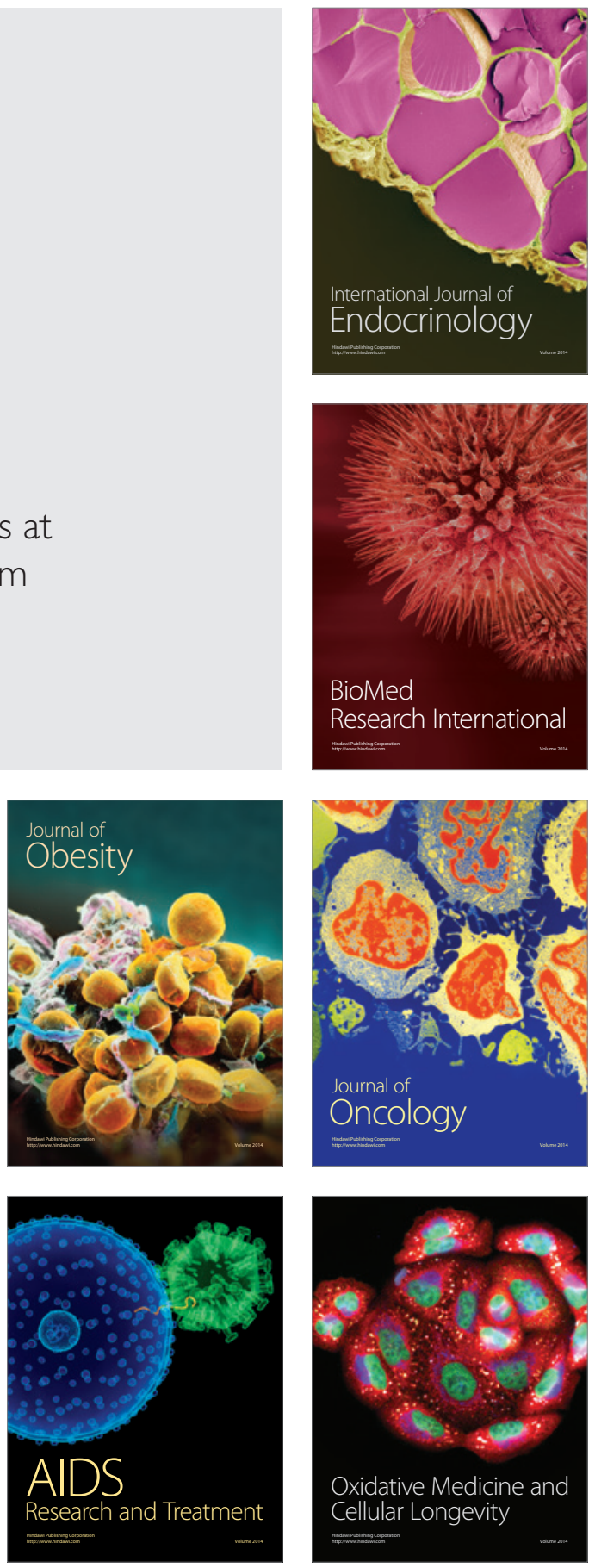